\title{
Assessment of Genetic Variability in Cucumber (Cucumis sativus L.)
}

\author{
Chandan Singh Ahirwar* and D.K. Singh \\ Department of Vegetable Science, G.B. Pant University of Agriculture and Technology, \\ Pantnagar, (Uttarakhand)-263145, India \\ *Corresponding author
}

\begin{tabular}{|c|}
\hline Keywords \\
\hline $\begin{array}{l}\text { Cucumber (Cucumis } \\
\text { sativus L.), } \\
\text { Variability, PCV, } \\
\text { GCV, Heritability, } \\
\text { Genetic advance }\end{array}$ \\
\hline Article Info \\
\hline $\begin{array}{l}\text { Accepted: } \\
\text { 07 February } 2018 \\
\text { Available Online: } \\
10 \text { March } 2018\end{array}$ \\
\hline
\end{tabular}

\section{Introduction}

Cucurbits (family Cucurbitaceae) are frostsensitive, predominantly tendril-bearing vines,

\section{A B S T R A C T}

The present experiment was carried out at vegetable research center, G B Pant University of Agriculture and Technology, Pantnagar, Uttarakhand during the July-October, 2014 and February-June, 2015 in randomized block design with three replications to assess genetic variability, heritability and genetic advance as percent of mean for various yield and its contributing traits. data were recorded on days to first male flowers, node number to first male flower, days to first female flowers, node number to first female flower, internodal length, days to first fruit harvest, number of fruits per plant, fruit length, fruit diameter, fruit weight, test weight, seed index, primary branches per plant, plant height total fruit yield per hectare. Analysis of variance revealed significant differences among the genotypes for all the traits studied indicating the presence of sufficient variability in the studied material. The phenotypic coefficient of variation (PCV) was higher than genotypic coefficient of variation (GCV) and the difference between PCV and GCV was narrow for most of the characters revealing little influence of the environment in the expression of these traits. During first season, phenotypic coefficient (PCV) of variation was highest for fruit length (32.52), in second season, was highest for yield (38.58) and pooled analysis was highest for yield (33.50) was recorded. During first season, genotypic coefficient (GCV) of variation was highest for fruit length (30.04), in second season, was highest for yield (38.47) and pooled analysis was found moderate for characters, namely, fruit length (27.65) was recorded. The range of heritability in broad sense varied from days to first male flowers (40.91) to fruit weight (98.51) in first season, yield (99.44) to seed index (67.18) in second season and in pooled analysis fruit weight $(\mathrm{g})$ (88.85) to primary branches per plant (25.31). Genetic advance as percentage of mean were found highest for fruit length (57.16), yield (79.03) and highest for fruit length (52.15) in first season, second season and pooled analysis data was recorded respectively. It may be concluded that the existence of wide range of genetic variability in the genotypes for these traits. revealed these traits are under the control of additive gene action and lower influence of environmental factor in the expression of these traits with possibility for genetic improvement through simple selection.

which are found in subtropical and tropical regions around the globe (Robinson and Decker-Walters, 1999). India is blessed with a rich diversity of cucurbits and is believed to be 
the primary and secondary centers of origin of many of the gourds and melons (Choudhury, 1996). Gourds, melons, squashes and cucumbers are the main group of crops under the family Cucurbitaceae. Cucurbits (the Cucurbitaceae family) are composed of 118 genera and 825 species. Members of this family are distributed primarily in tropical and subtropical regions of the world (Wang, et al., 2007).

The most economically important cucurbits according to world total creation are watermelon (Citrullus lanatus), cucumber (Cucumis sativus) and melon (Cucumis melo) (FAO 2006). The Cucurbitaceae includes two subfamilies Zanonioideae and the Cucurbitoideae. Cucurbitoideae comprises eight tribes one of which is Melothrieae which includes the genus Cucumis. The genus includes 30 wild and cultivated types that are spread throughout the world and has two major species: cucumber and melon.

The subgenus Cucumis includes SinoHimalayan species like Cucumis sativus $(2 \mathrm{n}=$ $2 \mathrm{x}=14)$ and $C$. hystrix Chakr. $(2 \mathrm{n}=2 \mathrm{x}=24)$. The wild $C$. hystrixis only found in Yunnan province of Southern China and has unique genetic traits (Prohens and Nuez 2008). C. sativushas several botanical groups like var. sativus, the cultivated cucumber and var. hardwickii, the wild form. Commercial cucumber, mentioned to as Cucumis sativusis thought to have originated in the southern Himalayan foothills region of Asia. C. sativus var. hardwickii (Royle) Alef.is a wild freeliving variety of Cucumis s. var. sativus that can be seen in Himalayan foothills. Cucumber has a small chromosome complement with $\mathrm{n}=$ $\mathrm{x}=7$ and a small haploid genome of 367 $\mathrm{Mbp} / \mathrm{C}$. The plant possesses unique properties with its genome. The mitochondrial genome is the largest of all eukaryotes. Conversely cucumber has a narrow genetic immoral, with a genetic variability of only $3-8 \%$.

\section{Materials and Methods}

The present investigation was conducted during July-October, 2014 and February-June, 2015 at Vegetable Research Centre and NAIP laboratory, Department of Vegetable Science in G.B. Pant University of Agriculture and Technology, Pantnagar, Uttarakhand. Pantnagar is situated in the foot hills of Himalayan region (Shivalik hills) and falls under humid subtropical climate zone in narrow belt called Tarai. Geographically, Vegetable Research Centre is situated at the latitude of $29.5^{\circ} \mathrm{N}$, longitude $79.3^{\circ} \mathrm{E}$ and at an altitude of 243.84 meters above the mean sea level. Total 46 genotypes of cucumber (Cucumis sativus L.) were used as experimental material in present experiment. The genotypes were diverse with respect to morphological and important economical traits. The experiment was laid out in randomized block design with three replications. Healthy and uniform sowing of seeds was main field in plots with a spacing of 3 meters $\times 0.60 \mathrm{~cm}$ during the evening hours of during July-October, 2014 and FebruaryJune, 2015. The crops were grown with standard package of practices.

The observations on various growth, yield and qualitative characters viz. observed highly significant differences for all the traits under study. A wide range of variability along with estimates of PCV and GCV was observed for days to 1st female flower anthesis, number of primary branches per plant, number of fruits per plant, number of node bearing female flowers per plant, fruit length, fruit weight, cavity of fruit at edible stage and fruit yield per plant. High heritability and high expected genetic gain were observed for days to $1 \mathrm{st}$ female flower anthesis, number of primary branches per plant, number of fruits/plant, fruit length and fruit diameter, 100-seed weight, cavity of fruit at edible stage and fruit yield/plant. 


\section{Results and Discussion}

The estimation of variability parameter i.e. Phenotypic (PCV), Genotypic (GCV), and environmental (ECV) coefficient of variation for yield and other characters are presented in Table 1, 2 and 3 .

During first season, genotypic coefficient of variation was highest for fruit length (30.04) whereas lowest estimate of GCV was recorded for days to first female flowers (8.60). Characters such as fruit weight (14.79), primary branches/ plant (16.79), number of fruits per plant (17.68), internodal length (17.77), test weight (19.25), node number to first male flower (20.24), seed index (23.62), yield (24.11), plant height (25.90), fruit diameter (27.26) and node number to first female flower (27.46) showed moderate GCV values. The character viz., days to first fruit harvest (11.32) and days to first male flowers (9.01), exhibited significantly lower value of GCV.

In second season, genotypic coefficient of variation was highest for yield (38.47) followed by internodal length (33.94) and number of fruits per plant (33.35). Characters such as plant height (27.94), fruit length (26.74), node number to first female flower (27.46), primary branches/ plant (23.56), fruit diameter (23.07), node number to first male flower (22.09), seed index (20.13) days to first fruit harvest (15.92) and fruit weight (15.54) showed moderate heritability. Test weight (14.66), days to first female flowers (13.55) and days to first male flowers (9.77) exhibited significantly lower value for GCV.

In pooled analysis genotypic coefficient of variation was found moderate for characters, namely, fruit length (27.65) followed by yield (24.77), node number to first female flower (24.07), fruit diameter (21.22), internodal length (19.46), number of fruits per plant
(18.90), plant height (16.86), seed index (15.28), node number to first male flower (15.21). Characters, namely, fruit weight (14.38), primary branches/ plant (11.94), days to first female flowers (10.51), test weight (10.02), days to first male flowers (8.45) and days to first fruit harvest (8.28) exhibited significantly lower value for GCV.

During first season, phenotypic coefficient of variation was highest for fruit length (32.52) followed by node number to first female flower (30.72), whereas lowest estimate of PCV was recorded for days to first female flowers (11.83). Characters such as number of fruits per plant (18.50), test weight (19.62), primary branches/ plant (21.13), internodal length (23.36), yield (24.62), node number to first male flower (25.60), seed index (26.56), plant height (28.97) and fruit diameter (29.15) showed moderate PCV values. The character viz., fruit weight (14.91), days to first male flowers (14.09) and days to first fruit harvest (12.73) exhibited significantly lower value for PCV.

In second season, phenotypic coefficient of variation was highest for yield (38.58) followed by internodal length (34.53), number of fruits per plant (33.46) and plant height (30.79). Characters such as test weight (15.84), fruit weight (15.61), days to first fruit harvesting $(16.52$,) seed index (24.56), node number to first male flower (24.90), primary branches/ plant (26.52), fruit diameter (26.73), fruit length $(\mathrm{cm})(27.69)$ and node number to first female flower (29.19) showed moderate PCV values. The character viz., days to first female flowers (13.76) and days to first male flowers (10.14) exhibited significantly lower value for PCV.

In pooled analysis phenotypic coefficient of variation was highest for yield (33.50) followed by internodal length (33.08) and fruit length (30.20). Characters, namely, plant 
height (29.99), node number to first female flower (29.97), number of fruits per plant (28.39), fruit diameter (28.12), seed index (25.53), node number to first male flower (25.25), primary branches/ plant (23.73), test weight (17.44), days to first fruit harvest (15.35) and fruit weight (15.26) showed moderate PCV values. The character viz., days to first female flowers (12.90) and days to first male flowers (12.13) exhibited significantly lower value for $\mathrm{PCV}$.

PCV was slightly higher than GCV for all the traits that indicated that the characters were not influenced by environment effects. Rastogi and Arya (1990), Saikia et al., (1995), Karuppiah et al., (2002), Kumar et al., (2008) and Mehdi and Khan (2009) observed that the coefficients of genotypic and phenotypic variability were moderate to high for different characters in cucumber.

Variation at phenotypic level is a combination of genetic as well as environmental variability, with does not help in selection. Hence, the decisive factors primarily rest on genetic variability or more specifically, additive genetic variability in which a breeder is mostly interested. Statistics like range, mean coefficient of variation, heritability at phenotypic and genotypic advance provide basic information on the variation of a character at phenotypic and genotypic level. This also gives an indication of the influence of environment in bringing about the variation.

The phenotypic variation consist of genotypic and environmental variability and therefore, it does not necessary ensure effective selection because it may sometime be largely due to environmental influences. Genetic variability and more specifically the additive genetic variation is important for a plant breeder as it indicates positively, the genetic gain through selection.
Estimation of heritability, genetic advance and genetic advance as percent of mean

The estimation of heritability in broad sense, genetic advance and genetic advance as a percentage of mean are given in Table 4-6. The range of heritability in broad sense varied from days to first male flowers (40.91) to fruit weight (98.51) in first season, yield (99.44) to seed index (67.18) in second season and in pooled analysis fruit weight $(\mathrm{g})$ (88.85) to primary branches/plant (25.31).

In first season fruit weight (g) (98.51) showed highest percentage of heritability as compare to other characters followed by test weight (96.26), yield (95.91), number of fruits per plant (91.35), fruit diameter (87.47), fruit length (85.33). Characters, mainly, plant height (79.94), node number to first female flower (79.92), seed index (79.08) and days to first fruits harvest (79.11) showed moderate value of heritability.

Characters, namely, primary branches/ plant (63.11) node number to first male flower (62.47), internodal length (57.84), days to first female flowers (52.88) and days to first male flowers (40.91) showed lower value of heritability.

In second season yield (99.44) showed highest percentage of heritability as compare to other characters followed by no. of fruits per plant (99.33), fruit weight $(99.11)$, days to first female flowers (96.94), internodal length (96.56), fruit length (93.24), days to first fruit harvest (92.81), days to first male flowers (92.76), test weight (85.56) and plant height (82.35).Characters namely primary branches/ plant (78.92), node number to first male flower (78.75), node number to first female flower (77.64), fruit diameter (74.47) and seed index (67.18) showed moderate value of heritability. 
Table.1 Analysis of variance for yield and its contributing traits in cucumber (first season)

\begin{tabular}{|c|c|c|c|c|c|c|c|c|c|c|c|c|c|c|c|c|}
\hline \multirow[b]{2}{*}{$\begin{array}{l}\text { Source of } \\
\text { variation }\end{array}$} & \multirow[b]{2}{*}{$\begin{array}{l}\text { Degree of } \\
\text { freedom }\end{array}$} & \multicolumn{15}{|c|}{ Mean sum of squares } \\
\hline & & $\begin{array}{l}\text { Days to } \\
\text { first male } \\
\text { flower }\end{array}$ & $\begin{array}{c}\text { Node } \\
\text { number to } \\
\text { first male } \\
\text { flower }\end{array}$ & $\begin{array}{c}\text { Days to } \\
\text { first } \\
\text { female } \\
\text { flower }\end{array}$ & $\begin{array}{c}\text { Node number to } \\
\text { first } \\
\text { femaleflower }\end{array}$ & $\begin{array}{l}\text { Internodal } \\
\text { length }(\mathbf{c m})\end{array}$ & $\begin{array}{c}\text { Days to } \\
\text { first fruit } \\
\text { harvest }\end{array}$ & $\begin{array}{c}\text { Number of } \\
\text { fruits per } \\
\text { plant }\end{array}$ & $\begin{array}{c}\text { Fruit } \\
\text { length } \\
(\mathrm{cm})\end{array}$ & $\begin{array}{c}\text { Fruit } \\
\text { diameter } \\
(\mathbf{c m})\end{array}$ & $\begin{array}{c}\text { Fruit } \\
\text { weight }(\mathrm{g})\end{array}$ & $\begin{array}{l}\text { Test } \\
\text { weight } \\
\text { (gm.) }\end{array}$ & $\begin{array}{l}\text { Seed } \\
\text { Index } \\
(\mathrm{gm} .)\end{array}$ & $\begin{array}{c}\text { Primary } \\
\text { branches/ } \\
\text { Plant }\end{array}$ & $\begin{array}{c}\text { Plant } \\
\text { height } \\
(\mathbf{m} .)\end{array}$ & $\begin{array}{l}\text { Yield } \\
\text { (q/ha) }\end{array}$ \\
\hline Replication & 2 & 10.91 & 1.83 & 1.66 & 0.21 & 0.03 & 0.07 & 0.13 & 3.46 & 0.15 & 23.81 & 0.72 & 0.03 & 0.58 & 0.03 & 51.63 \\
\hline Treatment & 45 & $52.46^{* *}$ & 4.29 & $53.91 * *$ & $12.12 *$ & 4.28 & $60.49^{* *}$ & 3.04 & $75.33^{* *}$ & 3.51 & $5469.69 * *$ & $45.19 *$ & 1.85 & $2.88^{*}$ & 1.01 & $1426.77 * *$ \\
\hline Error & 90 & 17.05 & 0.72 & 12.35 & 0.94 & 0.84 & 4.89 & 0.09 & 4.09 & 0.16 & 27.36 & 0.58 & 0.15 & 0.47 & 0.08 & 19.99 \\
\hline
\end{tabular}

Table.2 Analysis of variance for yield and its contributing traits in cucumber (second season)

\begin{tabular}{|c|c|c|c|c|c|c|c|c|c|c|c|c|c|c|c|c|}
\hline \multirow[b]{2}{*}{$\begin{array}{l}\text { Source of } \\
\text { variation }\end{array}$} & \multirow[b]{2}{*}{$\begin{array}{l}\text { Degree } \\
\text { of } \\
\text { freedom }\end{array}$} & \multicolumn{15}{|c|}{ Mean sum of squares } \\
\hline & & $\begin{array}{c}\text { Days to } \\
\text { first } \\
\text { male } \\
\text { flower }\end{array}$ & $\begin{array}{c}\text { Node } \\
\text { number } \\
\text { to first } \\
\text { male } \\
\text { flower }\end{array}$ & $\begin{array}{l}\text { Days to } \\
\text { first } \\
\text { female } \\
\text { flower }\end{array}$ & $\begin{array}{c}\text { Node } \\
\text { number to } \\
\text { first } \\
\text { femaleflower }\end{array}$ & $\begin{array}{l}\text { Internodal } \\
\text { length } \\
\text { (cm) }\end{array}$ & $\begin{array}{c}\text { Days to } \\
\text { first } \\
\text { fruit } \\
\text { harvest }\end{array}$ & $\begin{array}{c}\text { Number } \\
\text { of fruits } \\
\text { per } \\
\text { plant }\end{array}$ & $\begin{array}{c}\text { Fruit } \\
\text { length } \\
\text { (cm) }\end{array}$ & $\begin{array}{l}\text { Fruit } \\
\text { diameter } \\
(\mathbf{c m})\end{array}$ & $\begin{array}{c}\text { Fruit } \\
\text { weight (g) }\end{array}$ & $\begin{array}{c}\text { Test } \\
\text { weight } \\
\text { (gm.) }\end{array}$ & $\begin{array}{l}\text { Seed } \\
\text { Index } \\
\text { (gm.) }\end{array}$ & $\begin{array}{c}\text { Primary } \\
\text { branches/ } \\
\text { Plant }\end{array}$ & $\begin{array}{c}\text { Plant } \\
\text { height } \\
(\mathbf{m} .)\end{array}$ & $\begin{array}{l}\text { Yield } \\
\text { (q/ha) }\end{array}$ \\
\hline Replication & 2 & 3.88 & 0.12 & 1.44 & 0.46 & 0.35 & 9.95 & 0.02 & 2.15 & 0.36 & 9.09 & 4.57 & 0.01 & 0.27 & 0.04 & 1.91 \\
\hline Treatment & 45 & $49.61 * *$ & 4.73 & $118.04 * *$ & 10.79 & $36.59 *$ & $190.33 * *$ & $14.99 *$ & $57.97 * *$ & 2.19 & $5770.65^{* *}$ & $69.09 *$ & 1.50 & $4.19^{*}$ & 1.36 & $4895.29 * *$ \\
\hline Error & 90 & 1.26 & 0.39 & 1.24 & 0.95 & 0.43 & 4.79 & 0.04 & 1.37 & 0.23 & 17.19 & 3.68 & 0.23 & 0.35 & 0.09 & 9.14 \\
\hline
\end{tabular}

Table.3 Analysis of variance for yield and its contributing traits in cucumber (Pooled)

\begin{tabular}{|c|c|c|c|c|c|c|c|c|c|c|c|c|c|c|c|c|}
\hline \multirow{2}{*}{$\begin{array}{l}\text { Source of } \\
\text { variation }\end{array}$} & \multirow{2}{*}{$\begin{array}{c}\begin{array}{c}\text { Degree } \\
\text { of } \\
\text { freedom }\end{array} \\
\end{array}$} & \multicolumn{15}{|c|}{ Mean sum of squares } \\
\hline & & $\begin{array}{c}\text { Days to } \\
\text { first } \\
\text { male } \\
\text { flower }\end{array}$ & $\begin{array}{c}\text { Node } \\
\text { number } \\
\text { to first } \\
\text { male } \\
\text { flower }\end{array}$ & $\begin{array}{c}\text { Days to } \\
\text { first } \\
\text { female } \\
\text { flower }\end{array}$ & $\begin{array}{l}\text { Node } \\
\text { number } \\
\text { to first } \\
\text { female } \\
\text { flower }\end{array}$ & $\begin{array}{l}\text { Internodal } \\
\text { length } \\
\text { (cm) }\end{array}$ & $\begin{array}{c}\text { Days to } \\
\text { first } \\
\text { fruit } \\
\text { harvest }\end{array}$ & $\begin{array}{c}\text { Number } \\
\text { of fruits } \\
\text { per } \\
\text { plant }\end{array}$ & $\begin{array}{l}\text { Fruit } \\
\text { length } \\
\text { (cm) }\end{array}$ & $\begin{array}{c}\text { Fruit } \\
\text { diameter } \\
\text { (cm) }\end{array}$ & $\begin{array}{c}\text { Fruit } \\
\text { weight }(g)\end{array}$ & $\begin{array}{c}\text { Test } \\
\text { weight } \\
\text { (gm.) }\end{array}$ & $\begin{array}{l}\text { Seed } \\
\text { Index } \\
\text { (gm.) }\end{array}$ & $\begin{array}{c}\text { Primary } \\
\text { branches/ } \\
\text { Plant }\end{array}$ & $\begin{array}{c}\text { Plant } \\
\text { height } \\
(\mathbf{m} .)\end{array}$ & $\begin{array}{l}\text { Yield } \\
\text { (q/ha) }\end{array}$ \\
\hline Replication & 2 & 3.02 & 0.54 & 2.34 & 0.49 & 0.19 & 5.83 & 0.03 & 0.09 & 0.23 & 4.13 & 3.18 & 0.019 & 0.05 & 0.06 & 16.87 \\
\hline Treatment & 45 & $79.09^{* *}$ & 5.27 & $143.31 * *$ & $18.792 *$ & $19.75^{*}$ & $110.49^{* *}$ & $9.79^{*}$ & $124.79 * *$ & 4.15 & $10276.43^{* *}$ & $54.28 *$ & 1.95 & $3.28^{*}$ & 1.17 & $3971.96 * *$ \\
\hline Error & 90 & 11.92 & 1.19 & 11.16 & 1.58 & 4.74 & 31.94 & 1.69 & 3.89 & 0.47 & 210.59 & 13.71 & 0.45 & 1.09 & 0.31 & 481.67 \\
\hline
\end{tabular}

*= Significant at $5 \%$ level of significance

$* *=$ Significant at $1 \%$ level of significance 
Table.4 Estimation of coefficient of variation and genetic parameters in cucumber (first season)

\begin{tabular}{|c|c|c|c|c|c|c|c|c|c|}
\hline \multirow[t]{2}{*}{ S. No. } & \multirow[t]{2}{*}{ Characters } & \multirow{2}{*}{$\begin{array}{c}\text { General } \\
\text { mean }\end{array}$} & \multirow[t]{2}{*}{ Range } & \multicolumn{3}{|c|}{ Coefficient of variation } & \multirow{2}{*}{$\begin{array}{c}\text { Heritability } \\
(\%)\end{array}$} & \multirow{2}{*}{$\begin{array}{l}\text { Genetic } \\
\text { advance }\end{array}$} & \multirow{2}{*}{$\begin{array}{c}\text { G.A. as } \% \text { o } \\
\text { mean }\end{array}$} \\
\hline & & & & GCV & PCV & ECV & & & \\
\hline 1. & Days to first male flowers & 38.13 & $28.87-47.43$ & 9.01 & 14.09 & 10.83 & 40.91 & 4.53 & 11.87 \\
\hline 2. & Node number to first male flower & 5.39 & $2.33-7.66$ & 20.24 & 25.60 & 5.68 & 62.47 & 1.78 & 32.95 \\
\hline 3. & Days to first female flowers & 43.27 & $35.19-52.30$ & 8.60 & 11.83 & 8.12 & 52.88 & 5.58 & 12.88 \\
\hline 4. & Node number to first female flower & 7.03 & $3.00-11.00$ & 27.46 & 30.72 & 13.77 & 79.92 & 3.56 & 50.58 \\
\hline 5. & Internodal length $(\mathbf{c m})$ & 6.03 & $4.00-8.00$ & 17.77 & 23.36 & 15.17 & 57.84 & 1.68 & 27.84 \\
\hline 6. & Days to first fruit harvest & 38.03 & $29.46-49.80$ & 11.32 & 12.73 & 5.82 & 79.11 & 7.89 & 20.74 \\
\hline 7. & Number of fruits per plant & 5.60 & $3.30-8.30$ & 17.68 & 18.50 & 5.44 & 91.35 & 1.95 & 34.82 \\
\hline 8. & Fruit length $(\mathrm{cm})$ & 16.22 & $6.43-25.28$ & 30.04 & 32.52 & 12.46 & 85.33 & 9.27 & 57.16 \\
\hline 9. & Fruit diameter $(\mathbf{c m})$ & 3.87 & $2.25-8.57$ & 27.26 & 29.15 & 10.32 & 87.47 & 2.03 & 52.52 \\
\hline 10. & Fruit weight (g) & 287.89 & $182.98-371.87$ & 14.79 & 14.91 & 1.82 & 98.51 & 87.09 & 30.25 \\
\hline 11. & Test weight (gm.) & 20.03 & $10.73-27.48$ & 19.25 & 19.62 & 3.80 & 96.26 & 7.79 & 38.91 \\
\hline 12. & Seed Index (gm.) & 3.18 & $1.61-5.65$ & 23.62 & 26.56 & 12.15 & 79.08 & 1.38 & 43.26 \\
\hline 13. & Primary branches/ Plant & 5.33 & $3.00-7.66$ & 16.79 & 21.13 & 12.84 & 63.11 & 1.47 & 27.47 \\
\hline 14. & Plant height (met.) & 2.15 & $1.33-3.80$ & 25.90 & 28.97 & 12.98 & 79.94 & 1.03 & 47.71 \\
\hline 15. & Yield (Q/ha) & 89.81 & $48.80-144.48$ & 24.11 & 24.62 & 4.98 & 95.91 & 43.69 & 48.65 \\
\hline
\end{tabular}

Table.5 Estimation of coefficient of variation and genetic parameters in cucumber (second season)

\begin{tabular}{|c|c|c|c|c|c|c|c|c|c|}
\hline \multirow[t]{2}{*}{ S. No. } & \multirow[t]{2}{*}{ Characters } & \multirow{2}{*}{$\begin{array}{c}\text { General } \\
\text { mean }\end{array}$} & \multirow[t]{2}{*}{ Range } & \multicolumn{3}{|c|}{ Coefficient of variation } & \multirow{2}{*}{$\begin{array}{l}\text { Heritability } \\
(\%)\end{array}$} & \multirow{2}{*}{$\begin{array}{l}\text { Genetic } \\
\text { advance }\end{array}$} & \multirow{2}{*}{$\begin{array}{c}\text { G.A. as \% } \\
\text { of mean }\end{array}$} \\
\hline & & & & GCV & PCV & ECV & & & \\
\hline 1. & Days to first male flowers & 41.11 & $31.76-51.13$ & 9.77 & 10.14 & 2.73 & 92.76 & 7.96 & 19.38 \\
\hline 2. & Node number to first male flower & 5.44 & $2.33-7.66$ & 22.09 & 24.90 & 11.48 & 78.75 & 2.20 & 40.39 \\
\hline 3. & Days to first female flowers & 46.05 & $30.76-55.70$ & 13.55 & 13.76 & 2.41 & 96.94 & 12.66 & 27.48 \\
\hline 4. & Node number to first female flower & 7.04 & $1.00-9.00$ & 25.72 & 29.19 & 13.80 & 77.64 & 3.29 & 46.69 \\
\hline 5. & Internodal length $(\mathrm{cm})$ & 10.23 & $4.28-15.73$ & 33.94 & 34.53 & 6.40 & 96.56 & 7.03 & 68.70 \\
\hline 6. & Days to first fruit harvest & 49.41 & $35.48-67.35$ & 15.92 & 16.52 & 4.43 & 92.81 & 15.61 & 31.59 \\
\hline 7. & No. of fruits per plant & 6.69 & $2.80-10.56$ & 33.35 & 33.46 & 2.73 & 99.33 & 4.58 & 68.47 \\
\hline 8. & Fruit length $(\mathrm{cm})$ & 16.25 & $6.06-25.26$ & 26.74 & 27.69 & 7.20 & 93.24 & 8.64 & 53.18 \\
\hline 9. & Fruit diameter (cm) & 3.50 & 2.24- 5.14 & 23.07 & 26.73 & 13.51 & 74.47 & 1.44 & 41.01 \\
\hline 10. & Fruit weight (g) & 281.75 & $180.16-380.11$ & 15.54 & 15.61 & 1.47 & 99.11 & 89.81 & 31.88 \\
\hline 11. & Test weight (gm.) & 31.86 & $19.50-40.70$ & 14.66 & 15.84 & 6.02 & 85.56 & 8.90 & 27.93 \\
\hline 12. & Seed Index (gm.) & 3.35 & 2.10- 4.59 & 20.13 & 24.56 & 14.07 & 67.18 & 1.14 & 33.98 \\
\hline 13. & Primary branches/ Plant & 4.80 & $2.00-7.66$ & 23.56 & 26.52 & 12.18 & 78.92 & 2.07 & 43.12 \\
\hline 14. & Plant height (met.) & 2.33 & 1.16- 3.66 & 27.94 & 30.79 & 12.94 & 82.35 & 1.22 & 52.24 \\
\hline 15. & Yield (Q/ha) & 104.90 & $46.30-202.00$ & 38.47 & 38.58 & 2.88 & 99.44 & 82.90 & 79.03 \\
\hline
\end{tabular}


Table.6 Estimation of coefficient of variation and genetic parameters in cucumber (pooled)

\begin{tabular}{|c|c|c|c|c|c|c|c|c|c|}
\hline \multirow{2}{*}{$\begin{array}{l}\text { S. } \\
\text { No. }\end{array}$} & \multirow[t]{2}{*}{ Characters } & \multirow{2}{*}{$\begin{array}{l}\text { General } \\
\text { mean }\end{array}$} & \multirow[t]{2}{*}{ Range } & \multicolumn{3}{|c|}{ Coefficient of variation } & \multirow{2}{*}{$\begin{array}{l}\text { Heritability } \\
\qquad(\%)\end{array}$} & \multirow{2}{*}{$\begin{array}{l}\text { Genetic } \\
\text { advance }\end{array}$} & \multirow{2}{*}{$\begin{array}{c}\text { G.A. as \% } \\
\text { of mean }\end{array}$} \\
\hline & & & & GCV & PCV & ECV & & & \\
\hline 1. & Days to first male flowers & 39.62 & $\begin{array}{l}30.32- \\
46.68\end{array}$ & 8.45 & 12.13 & 8.71 & 48.44 & 4.80 & 12.11 \\
\hline 2. & $\begin{array}{l}\text { Node number to first male } \\
\text { flower }\end{array}$ & 5.42 & $3.33-7.50$ & 15.21 & 25.25 & 20.15 & 36.29 & 1.02 & 18.88 \\
\hline 3. & Days to first female flowers & 44.66 & $\begin{array}{l}33.78- \\
53.35\end{array}$ & 10.51 & 12.90 & 7.48 & 66.37 & 7.88 & 17.64 \\
\hline 4. & $\begin{array}{l}\text { Node number to first female } \\
\text { flower }\end{array}$ & 7.04 & $2.33-9.50$ & 24.07 & 29.97 & 17.84 & 64.54 & 2.80 & 39.84 \\
\hline 5. & Internodal length (cm) & 8.13 & $4.62-11.27$ & 19.46 & 33.08 & 26.75 & 34.60 & 1.92 & 23.58 \\
\hline 6. & Days to first fruit harvest & 43.72 & $\begin{array}{l}35.01- \\
55.05\end{array}$ & 8.28 & 15.35 & 12.93 & 29.07 & 4.02 & 9.19 \\
\hline 7. & No. of fruits per plant & 6.15 & $4.00-8.78$ & 18.90 & 28.39 & 21.18 & 44.34 & 1.59 & 25.93 \\
\hline 8. & Fruit length (cm) & 16.24 & $6.42-23.68$ & 27.65 & 30.20 & 12.14 & 83.84 & 8.47 & 52.15 \\
\hline 9. & Fruit diameter (cm) & 3.69 & $2.44-5.48$ & 21.22 & 28.12 & 18.45 & 56.96 & 1.22 & 33.00 \\
\hline 10. & Fruit weight (g) & 284.82 & $\begin{array}{l}181.57- \\
355.73\end{array}$ & 14.38 & 15.26 & 5.10 & 88.85 & 79.53 & 27.92 \\
\hline 11. & Test weight (gm.) & 25.95 & $\begin{array}{l}17.43- \\
30.19\end{array}$ & 10.02 & 17.44 & 14.27 & 33.05 & 3.08 & 11.87 \\
\hline 12. & Seed Index (gm.) & 3.27 & $2.42-4.90$ & 15.28 & 25.53 & 20.46 & 35.79 & 0.62 & 18.83 \\
\hline 13. & Primary branches/ Plant & 5.07 & $3.50-6.66$ & 11.94 & 23.73 & 20.51 & 25.31 & 0.63 & 12.37 \\
\hline 14. & Plant height (met.) & 2.24 & $1.47-3.20$ & 16.86 & 29.99 & 24.81 & 31.59 & 0.44 & 19.51 \\
\hline 15. & Yield (Q/ha) & 97.35 & $\begin{array}{l}60.56- \\
173.24\end{array}$ & 24.77 & 33.50 & 22.54 & 54.70 & 36.75 & 37.75 \\
\hline
\end{tabular}


However in pooled analysis fruit weight (88.85) showed maximum heritability followed by fruit length (83.84). Characters namely days to first female flowers (66.37), node number to first female flower (64.54), fruit diameter (56.96) and yield (54.70) showed moderately heritability. Days to first male flowers (48.44), number of fruits per plant (44.34), node number to first male flower (36.29), seed index (35.79), internodal length (34.60), test weight (33.05), plant height (31.59), days to first fruit harvest (29.07) and primary branches/ plant (25.31) were recorded for low value of heritability.

Genetic advance is the improvement over the base population that can potentially by make from selection for a character. It is function of the heritability of the amount of phenotypic variation and the selection differential that is used by breeder. The genetic advance depends on the extent of genetic variability, the magnitude of masking effect of genetic expression (environment influence) and the intensity of selection.

In the first season genetic advance as percentage of mean were found highest for fruit length (57.16) followed by fruit diameter (52.52), node number to first female flower (50.58), yield (48.65), plant height (47.71), seed index (43.26), test weight (38.91), number of fruits per plant (34.82), node number to first male flower (32.95) and fruit weight (30.25). Characters namely internodal length (27.84), primary branches/ plant (27.47), days to first fruit harvest (20.74) showed moderate value of genetic advance and days to first female flowers (12.88) and days to first male flowers (11.87) were recorded for low value of genetic advance as percentage of mean.

In second season genetic advance as percentage of mean were found highest for yield (79.03) followed by internodal length
(68.70), number of fruits per plant (68.47), fruit length (53.18), plant height (52.24), node number to first female flower (46.69), primary branches/ plant (43.12), fruit diameter (41.01), node number to first male flower (40.39), seed index (33.98), fruit weight (31.88), days to first fruit harvest (31.59). Characters, namely, test weight (27.93), days to first female flowers (27.48) and days to first male flowers (19.38) were recorded for moderate genetic advance as percentage of mean.

However in pooled analysis genetic advance as percentage of mean were found highest for fruit length (52.15) followed by node number to first female flower (39.84), yield (37.75), fruit diameter (33.00). Character namely fruit weight (27.92), number of fruits per plant (25.93), internodal length (23.58), and plant height (19.51), node number to first male flower (18.88), seed index (18.83) and days to first female flowers (17.64). Characters, namely, primary branches per plant (12.37), days to first male flowers (12.11), test weight (11.87) and days to first fruit harvest (9.19) were recorded for low value of genetic advance as percentage of mean.

Characters showing high heritability coupled with high genetic advances as per cent of mean were node number to first male flowers, node number to first female flowers, plant height and number of fruits per plant. Kumar et al., (2008) recoded high estimate of heritability along with genetic advance for all trait studied. Yadav et al., (2009) recorded high heritability and genetic advance for some traits. Mehdi and Khan (2009) reported that high heritability along with high genetic advance revealing that these characters are controlled by additive gene.

Heritability is the transmutability of characters from parents to offspring. In broad sense, it is the ratio of genotypic variance to 
phenotypic variance in percentage. The knowledge about the beneficial parameter would be useful to increase the efficiency of a breeding system since; it is a measure of success in separating genotypes by selection.

Analysis of variance revealed the presence of considerable amount of genetic variability for yield and its components studied in all the environments. The genotypes expressed high genotypic and phenotypic coefficient of variation, heritability (broad sense) accompanied with high value of genetic advance for number of fruits per plant, fruit weight, Fruit diameter $(\mathrm{cm})$, days to first fruit harvest, fruit length, fruit diameter, primary branches per plant, plant height total fruit yield per hectare etc. revealed these traits are under the control of additive gene action and lower influence of environmental factor in the expression of these traits with possibility for further improvement of these character through simple selection.

\section{Acknowledgment}

Authors are thankful to the administrative team and all the supporting staff involved in the present research especially Dr. M. L. Kushwaha and Dr. C.P. Singh. Department of Vegetable Science and Department of Horticulture, G.B. Pant University of Agriculture and Technology, Pantnagar (Uttarakhand), India for valuable suggestions and support during entire research work

\section{References}

Choudhary, F. M., Khlkhar, K. M., Jeelani, G., Ullah, H. and Riaz, S. 2004. Performance of some cucumber hybrids/lines under plastic tunnel. Sarhad Journal of Agriculture, 19 (4): 493-495.

Choudhary, M. L., Joshi, S. and Singh, A. 1985. Genetic studies in cucumber
(Cucumis sativus L.) Prog. Hort. 17(3): 236-240

Choudhury, B., 1996. Vegetables. National Book Trust, New Delhi, India.

Grubben, G.J. H. and Denton, O.A. 2004.Plant Resources of Tropcal Africa 2.Vegetable. PROTA Foundation, Wageningen; Backhuys, Leiden; CTA, Wageningen. 4: 103-111

Gulam -ud - Din; Ahmed, N., Narayan, R., Nzir, G. and Hussain, K. 2006. Variabkility studies in cucumber. Haryana J. of Hort. Sci. 35.3-4: 297298

Guseva, L.I. and Mospabn, M.V. 1984. Study of combing ability in the production of cucumber hybrids. Genetic heskieonso vyselektessi sel. Skohozyaistveny khrastenii I zhivothnykh (ed. By Zhuchenko, A.A.) pp. 28-29

Kumar, A., Kumar, S. And Pal, K. A. 2008.Genetic variability and character association for yield and yield traits in cucumber. Indian J. Hort. 65.4:423-428

Mehdi, M. and Khan, F. A. S. 2009. Variability and character association analysis in cucumber germplasm. J. Agri. \& Biological Res. 25.2:87-91

Phillips, R.L. 1999. Research needs in heterosis. In: Genetics and exploitation of heterosis in Crop. J.G. Coors and S. Pandey (eds.). pp 501-507.

Pierce L.C. and Brewbaker, J.L. 1973. Application of isozyme analysis in horticultural science. Hort. Scie., 8:1722.

Prohens, J., and Fernando Nuez, eds. 2008.Handbook of Plant breeding: Vegetables I: Asteraceae, Brassicaceae, Chenopodicaceae and Cucurbitaceae. Valencia, Spain. Universisad Politecnica de Valencia. http://www.springer.com (accessed June, 2009). 
Rastogi, K.B. and Arya, D. 1990. Variability studies in cucumber (Cucumis sativus L.). Veg. Sci. 17.2:224-226

Robinson, R.W. and Decker-Walters, D.S. 1999.Cucurbits. CAB International, Wallingford, Oxford, UK.

Wang, Y.H., Joobeur, T., Dean, R.A. and Staub, J. E. 2007.Cucurbits. Genome Mapping and Molecular Breeding in plants 5: Vegetables Whitehouse, R.N.H., Thompson, J.B. and Ribiero, M.A. M. 1958. Studies on the breeding of self pollinated creals. The use of diallel cross analysis in yield prediction. Euphytica 7:147-167
Yadav, Y. C., Kumar, S. and Singh, R. 2012. Studies on genetic variability, heritability and genetic advance in cucumber (Cucumis sativus L.). Hort. Flora Research Spectrum. 1(1): 34-37.

Yadav, Y. C., Kumar, S. and Singh, R. 2010.Correlation coefficient and path analysis in cucumber (Cucumis sativus L.). Prog. Hort. 42.1:71-75

Yadav, Y. C., Kumar, S., Bisen, B. and Dixit, S. K. 2009. Genetic variability, heritability and genetic advance for some traits in cucumber. Indian J. Hort. 66.4:488-491.

\section{How to cite this article:}

Chandan Singh Ahirwar and Singh, D.K. 2018. Assessment of Genetic Variability in Cucumber (Cucumis sativus L.). Int.J.Curr.Microbiol.App.Sci. 7(03): 813-822. doi: https://doi.org/10.20546/ijcmas.2018.703.095 